\title{
LATE STAGES OF STAR EVOLUTION
}

\author{
Foreword
}

Late stages of star evolution are now a topic of increasing interest because observations with higher and higher spectral and also angular resolutions are now possible in wide ranges of wavelengths. Concurrently, physical or semi-empirical models involving better and better input physics become available. However, comparison between what can be observed and what can be modelized is not as easy as for some other stages of evolution. This is still a problem and, in order to sum up our knowledge in the field, the French Physical Society, the Swiss Physical Society and the Swiss Society of Astrophysics and Astronomy decided to organise a joint symposium followed by some kind of enchiridion.

The publication of this "special issue" of the journal was decided as a sequel to this symposium organized in Lyon (France) in september 1989. We have gathered only review papers written later by people who had given invited talks.

Thus, in order to improve the interest and also the number of readers of this publication, we have asked for some additional work from the authors: instead of a simple development of their talk as for symposia proceedings, they have submitted review papers about the same topics; these papers have been criticized by referees, as usually. Thus, though this issue is only devoted to the topics discussed at Lyon, it contains a enchiridion in a domain on current study.

On behalf of the three societies, we thank the "ANNALES DE PHYSIQUE" and also the authors for their collaboration. 